\title{
Subject Index Vol. 15, 1997
}

Adhesion molecules 61 Ambulatory blood pressure monitoring 25 Amino acid 112

Anthropometry 25

Bacterial count 188 Bioimpedance 25 Bleeding time 84 Blood pressure 25

Interdialytic weight gain 25 Intradialytic parenteral nutrition 112

$\mathrm{Kt} / \mathrm{V} 169$

Limulus amebocyte lysate test 188 Low-density lipoprotein apheresis

182 Low dialysate flow rate 208 Lymphocytes 61

Chlorine dioxide 188 Cold dialysate 15 Conductivity kinetic model 34 Continuous ambulatory peritoneal dialysis 195

Desmopressin (DDAVP) 84 Dialysate 54 - temperature 15 Dialysis, adequacy 77 Dialyzer reuse 77

Endotoxin 188 Erythropoietin 112

Factor VIII complex 84 Fibrinogen 182 Fluid overload 25

Growth hormone 112

Malnutrition 112 Mathematical modelling 157 Monocytes 61

nPCR 169

Nutrition 77

On-line predilution hemofiltra-

tion 169 Oxygen tension 15

Paired filtration dialysis 34 Peritoneal dialysis 112 Plasma exchange 45 Plasmapheresis 45

Protein catabolic rate 157

Recombinant human erythropoietin 77 Residual renal function 157

Hemodiafiltration 34 Hemodialysis 15,25,61,77,92,

112,157,200 Hemorheology 182 Heparin-free hypothermal $\left(20^{\circ} \mathrm{C}\right)$

hemodialysis 200 Hepatitis B virus 195 - C virus 195 High-flux membrane 208

Hypercholesterolaemia treatment

182 Hypoxia 15

Immune system 61 Insulin-like growth factor-1 112

Sepsis 45

Sodium kinetic model 34

Sterilization 54

Tissue plasminogen activator 84

Ultrapure high-flux hemodialysis

169 Urea 92

- kinetic modelling 157 Uremia 84

Viscosity 182 
KAIUiER

(C) 1997 S. KargerAG, Basel

E-Mail karger@karger.ch Fax+ 41613061234 http://www.karger.ch 